\section{Volumetric Change Detection with using Structure from Motion - The Impact of Repeat Station Imaging}

Gl_Forum 2018, Issue 1 Page: 135 - 151 Full Paper Corresponding Author: aloerch@sdsu.edu DOI: 10.1553/giscience2018_01_s135

\author{
Andrew C. Loerch1, Gernot Paulus² and Christopher D. Lippitt' \\ 'University of New Mexico, USA \\ ${ }^{2}$ Carinthia University of Applied Sciences, Austria
}

\begin{abstract}
Repeat Station Imaging (RSI) for image acquisition is compared with non-RSI to assess the methods' effects on vertical and volumetric estimation using structure from motion (SFM). Aerial triangulation (i.e., SFM) is used to create three-dimensional reconstructions of the study area using unmanned aerial vehicle-acquired imagery. Targets of known volume were deployed throughout the scene and manipulated to create changes between the first and subsequent flights. An RSI flight and two non-RSI flights were compared to a baseline flight in order to estimate a series of introduced volumetric changes, which were then compared to known volume changes. Using images with a nominal ground sampling distance of $1.96 \mathrm{~cm}$, results show a total root-mean-squared-error (RMSE) of $0.035 \mathrm{~m}^{3}$ and mean percent error (MPE) of $25.9 \%$ for the RSI flight, and average RMSE of $0.057 \mathrm{~m}^{3}$ and MPE 33.3\% for the two non-RSI flights. For the measurement of volumetric changes to extant features, the RSI flight had an RMSE of $0.026 \mathrm{~m}^{3}$ and an MPE of $17.6 \%$; the average RMSE and MPE of the two non-RSI flights were $0.071 \mathrm{~m}^{3}$ and $39.4 \%$. These results show that RSI has the potential to improve the accuracy of volumetric and height change estimation.
\end{abstract}

\title{
Keywords:
}

structure from motion, change detection, unmanned aerial vehicle, digital surface model (abbreviations ${ }^{1}$ )

\section{Introduction}

Structure from motion (SFM), an automated technique for aerial triangulation, can be used to create digital surface models (DSM) of landscapes (Westoby et al., 2012). Modern improvements in platforms, digital cameras and software applications have made it possible to create these models with high spatial resolutions and high temporal frequency (Turner et

1 SFM: Structure from Motion, RSI: Repeat Station Imaging, UAS: Unmanned Aerial System, DEM: Digital Elevation Model, DSM: Digital Surface Model, PPK: Post-processing Kinematic, GNSS: Global Navigation Satellite System 
al., 2012; Westoby et al., 2012). Detecting changes to imaged landscapes is a primary focus of remote sensing, and the ready availability of high-resolution digital surface models could lend itself to the detection and measurement of volumetric change (Niethammer et al., 2012; Westoby et al., 2012). The use of the repeat station imaging (RSI) method has been demonstrated to improve the accuracy of co-registration between image pairs and, subsequently, detected changes (Coulter et al., 2003). The RSI approach replicates view geometry between time- 1 and time- $n$ acquisitions. Given that aerial triangulation relies fundamentally on view geometry to estimate height, we hypothesize that implementing RSI can improve the accuracy of volumetric change measurement from SFM by more closely matching the pattern of height estimate errors between time- 1 and time- $n$ DSMs.

\section{SFM for Volumetric Change}

Volumetric change measurements rely on the availability of DSMs, which represent the height and area of the surface of a scene (Westoby et al., 2012). SFM is a method of aerial triangulation used to create point clouds, similar to the first return from LiDAR, from which DSMs can be derived (Rosa and Stow, 2014; Westoby et al., 2012).

SFM can be used to create very dense point clouds and therefore very high resolution DSMs, following a generalized workflow. Images of a scene must be acquired with a large degree of overlap, ensuring both the presence of a large number of matching points between images and a large degree of variation in the view geometries of the features in a scene (Turner et al,, 2012; Westoby et al., 2012; Zhang et al., 2011). The step of aligning the imagery and creating a sparse point cloud is often referred to as 'bundle adjustment'; this step finds and matches points in imagery that are then used to estimate the internal and external orientations of the camera and align the images (Westoby et al., 2012). Once a bundle adjustment is completed, the images aligned and orientations of the camera known, a dense point cloud can be created and exported as a DSM (Westoby et al., 2012).

Measuring volumes and measuring volumetric changes from DSMs are not inherently the same process. Calculating the volumes of features in a DSM requires knowing the elevation of the surface on which the features exist (Jensen and Im, 2007; Niethammer et al., 2012). It is challenging to create accurate DEMs from dense point clouds, as noise in dense clouds leads to inaccurate interpolation of some surface values in the DEM (Turner et al., 2012). Where measuring volumetric change is a suitable alternative to measuring the absolute volumes of features, a baseline DSM can be subtracted from a time- $n$ DSM, and the resultant volumetric changes of features of interest can be measured by multiplying the area of a pixel by the changed height value of the pixel, and adding the results for all pixels in a feature (Jensen and Im, 2007).

\section{Traditional imagery acquisitions and Repeat Station Imaging}

Co-registration accuracy is a limiting factor in the accuracy of two-dimensional change detection (Song et al., 2001; Stow, 1999). The accuracy of change detections that utilize image-differencing is affected by the co-registration between images in a time series (Coulter et al., 2003; Lee et al., 2008; Stow, 1999). The method traditionally employed for acquiring imagery for aerial triangulation is the collection of flight lines spaced for a desired sidelap 
using a frame-based sensor designed to capture images based on either time intervals or distance intervals (Jensen and Im, 2007). This method of acquiring images ensures sufficient forward-lap and sidelap to ensure features are viewed from multiple perspectives and thus permit the estimation of height using parallax. While differential parallax between imagery in the same observation permits aerial triangulation, it makes co-registration between subsequent observations a challenge (Coulter et al., 2003; Stow, 1999). Parallax introduces variable relief displacement between observations (Slama et al., 1980). The distortions in vertical features and co-registration errors between time series resulting from varied view geometries reduce the accuracy of change detection results (Coulter et al., 2003; Stow, 1999; Stow et al., 2016).

Repeat Station Imaging is an alternative method for acquiring imagery for use in change detection. In RSI, the flight and imaging path is designed using GNSS waypoints, and the imaging sensor is triggered based on these waypoint stations (Coulter et al., 2003; Lippitt et al., 2015). Provided the same imaging sensor is used across acquisitions, the result is " multitemporal imagery with matched view geometry' (Lippitt et al., 2015). This method of image acquisition has been shown to result in horizontal spatial co-registrations between multitemporal image sets of between one and two pixels, even at high spatial resolutions (Coulter et al., 2003; Lippitt et al., 2015). Horizontal changes between image pairs and between two-dimensional orthomosaics have been the focus of existing literature on the use of RSI (Coulter et al., 2003; Lippitt et al., 2015).

Considering the change detection algorithms described by Jensen and Jung (Jensen and Im, 2007), which are reliant on accurate DEMs or DSMs that are either external to, or created from, the multitemporal image sets, it is logical to conclude that improvements to the accuracies of DEMs/DSMs should result in improvements in the accuracies of change detection methods that rely on the surface models. An outstanding question of RSI is whether repeated view geometries result in consistent parallax errors in feature positions and volumes over time.

\section{Material and Methods}

RSI was implemented with a fixed-wing UAS and PPK system to collect four time series of imagery: a baseline, a repeated view geometry image set (i.e., RSI), and two non-repeated view geometry image sets. Stacks of assembled cardboard boxes of known dimensions were used as the change objects. Agisoft Photoscan was used for the SFM image processing and ERDAS IMAGINE software was used to perform the change detections.

\section{Scene selection and preparation}

Figure 1 shows the study area, model airpark, surrounding region and principal experimental setup. The image used in the creation of the map in Figure 1 is an orthomosaic from the baseline flight. This area has three permanent ground control markers. At the time of the flights, the temperature was $28^{\circ}$ Celsius, the winds were calm to light on the ground, and there were no clouds directly over the study area. 
Boxes of varying sizes were deployed and manipulated between flights to introduce known volumetric changes. Individual boxes, once assembled, can be measured accurately and precisely, are lightweight, and can be quickly stacked and moved. To prevent stacked boxes from shifting due to slight breezes, they were duct-taped together when stacked. Twelve stacks of boxes at six different heights, and the UAS case, were deployed in the study area. Six stacks and the UAS case remained invariant throughout, and the other six stacks were manipulated by removing boxes to reduce their heights, or stacks were eliminated completely. Removed boxes were then deployed to create new objects. After manipulation, there were a total of nineteen deployed objects.

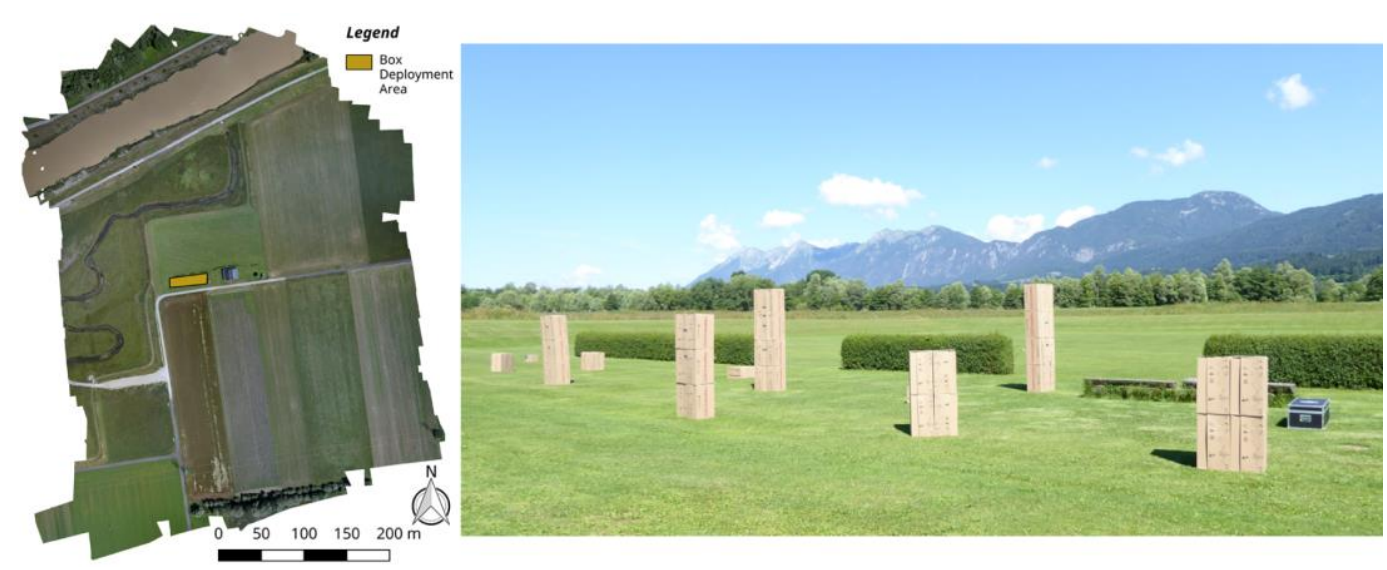

Figure 1: Location of the model airpark and study area at Feistritz an der Gail (left). Principal experimental setup of boxes (right)

Figure 2 shows the placement of the stacks. Stacks numbered 1 through 7 were deployed for the baseline. Stacks 8 through 13 are those newly added to the scene after the baseline flight. Finally, the letter ' $\mathrm{A}$ ' designates stacks that were changed after the baseline, and the letter ' $\mathrm{B}$ ' designates stacks that remained unchanged across all flights. Box $7 \mathrm{~B}$ is the only object used that was not a cardboard box; it is the UAS case. 


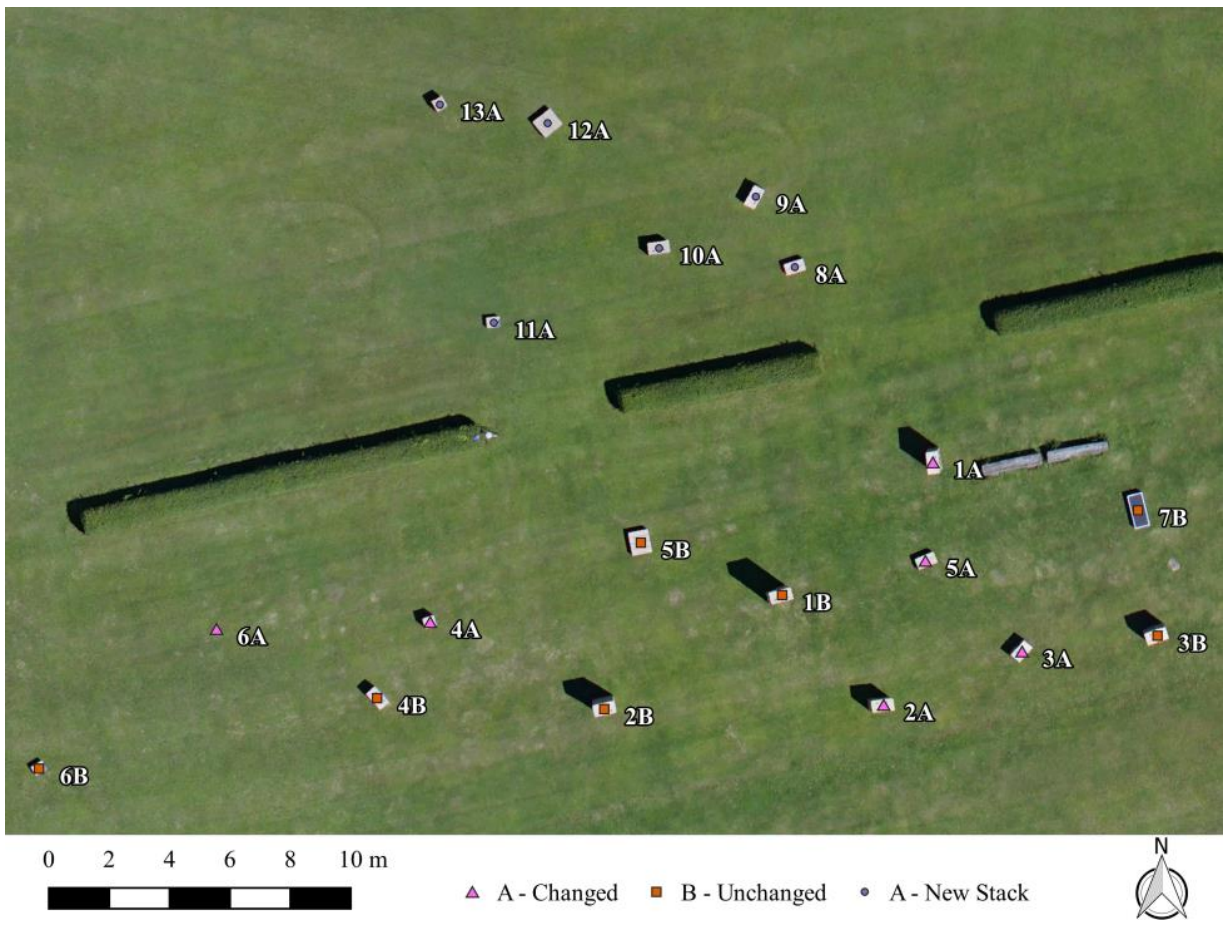

Figure 2: The final layout of box stacks post-baseline flight and volume changes

\section{Flight planning and image acquisition}

The C-Astral Bramor PPK fixed-wing UAS was chosen as the acquisition platform. The Bramor has a 2.5 hour flight time, can carry heavier, off-the-shelf digital cameras, and has a built-in PPK receiver that, used with a base station, achieves highly accurate post-processing locations of imagery (C-Astral, 2015). The specified absolute positional measurement accuracy, in post-processing, for this platform is $1.5 \mathrm{~cm}$. As tested, the positional accuracy achieved by this system for navigation was 4.22m RMSE. A Sony a6000 mirrorless camera with a $30 \mathrm{~mm}$ focal length lens was used as the sensor.

RSI-based acquisitions are intended to match image station positions in the sky over time in order to reduce differences in view geometries (Coulter et al., 2003). It is preferable and simplest to accomplish RSI using waypoints as camera stations and repeating those waypoints across acquisitions. The C-Astral Bramor, and many UAS and manned platforms, are designed to use waypoints for navigation but not camera triggering (C-Astral, 2015; Coulter et al., 2003). Four flights were made to configure and test the Bramor system for waypoint-based camera triggering; these resulted in horizontal RMSEs between repeated waypoints that were consistently greater than $5.5 \mathrm{~m}$, with waypoints often missed entirely.

As waypoint-based triggering for this UAS was determined through these tests to be unsatisfactory for RSI (Stow et al., 2016), all flight plans were designed using the time-based camera-triggering method. For the Bramor, this method works by initiating the cameratriggering at the beginning of each flight line, and triggering every $x$ number of seconds to 
achieve a specified overlap between images. To achieve RSI, the second flight plan was kept identical to the first. In the flight planning, this maintains the same heading, and the same horizontal and vertical locations where each image was captured between the initial and the second flights. Using this method, RSI was achieved between the baseline and second flights with a horizontal RMSE of $4.22 \mathrm{~m}$, and vertical RMSE of $0.77 \mathrm{~m}$. The defined horizontal velocity was $16 \mathrm{~m} / \mathrm{s}$ and the defined altitude was $150 \mathrm{~m}$. The designated overlap and sidelap were both $70 \%$, and the nominal ground sample distance was $1.96 \mathrm{~cm}$.

Flight 3 was designed so that the camera stations would be different from the baseline camera stations. This was accomplished by shifting the orientation of the flight lines by 30 degrees from the baseline flight lines. The minimum (closest) horizontal distance between camera stations from the initial flight and Flight 3 over the deployed objects was $5.25 \mathrm{~m}$, which is $1.03 \mathrm{~m}$ greater than the RMSE of the baseline and RSI flights' camera station locations, with an average heading difference of 30.64 degrees. The differences between the minimum distance and bearings of the camera stations' locations compared to the baseline show how Flight 3 differs from the RSI flight. The altitude was kept at $150 \mathrm{~m}$ for all flights to avoid differences in scale. Parameters for desired overlap and sidelap were replicated for all flights.

Flight 4 was also designed to introduce differences from the baseline camera stations' locations. This was accomplished by shifting the orientation of the flight lines by 60 degrees from the baseline flight lines. As with Flight 3, no attempt was made to match the horizontal locations where the images were captured. The minimum horizontal distance between camera stations from the baseline flight and Flight 4 over the deployed objects was $7.36 \mathrm{~m}$, which is 3.14 metres greater than the RMSE of the baseline and RSI flights' camera station locations, with an average heading difference of 55.2 degrees.

Figure 3 shows the three flights over the deployed objects, post-change, with the respective camera station locations. Although there is a noticeable difference between the initial flight paths and the RSI flight path, that difference is clearly less than for the third and fourth flights, as noted above. 
A) Flight 1 and Flight 2 (RSI)

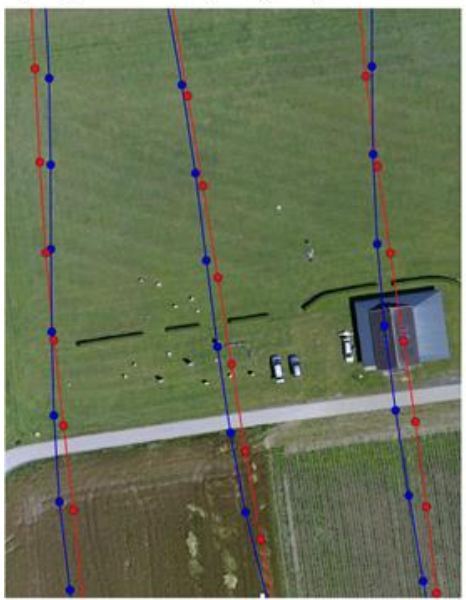

C) Flight 1 and Flight 4

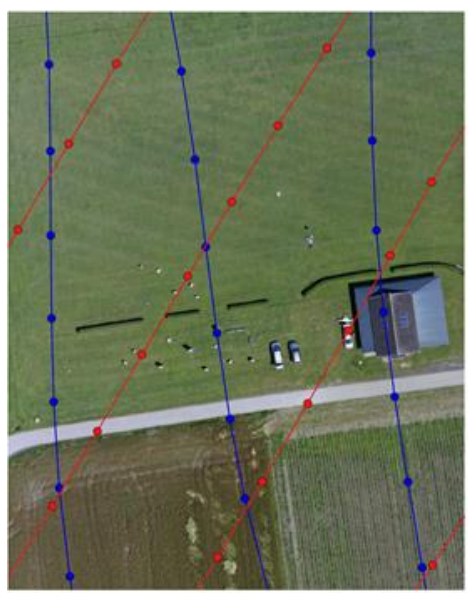

\section{B) Flight 1 and Flight 3}

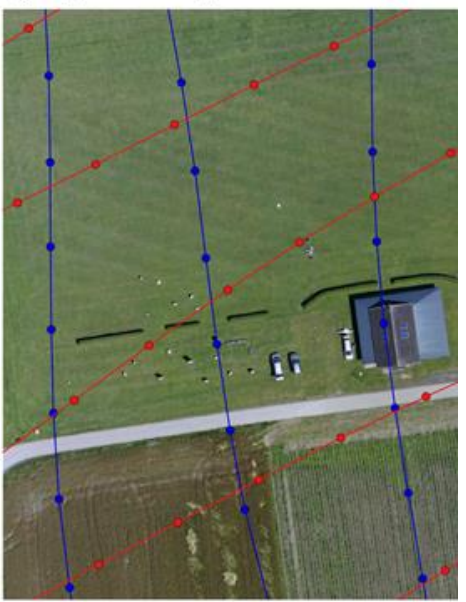

$\begin{array}{llllll}0 & 10 & 20 & 30 & 40 & 50\end{array}$

Legend

- Flight 1 (Baseline)

- Flight 1 Camera Station

- Post-Change Flight

- Post-Change Camera Station

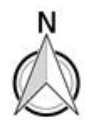

Figure 3: Flight Paths and Image Station Locations. A) Shows the adapted RSI method, B) shows flight path shifted by 30 degrees compared to original, C) shows flight path shifted 60 degrees compared to original

Parallax, or lean, of the stacks of boxes appears to change between flights, dependent on the incident view geometries. Figure 4 shows the appearance of parallax in two box stacks across the four flights. The stack on the left of the figure has a height of $0.659 \mathrm{~m}$, and the object on the right has a height of $1.977 \mathrm{~m}$. The parallax seen in the baseline flight is better reproduced in the RSI flight than in subsequent flights. 


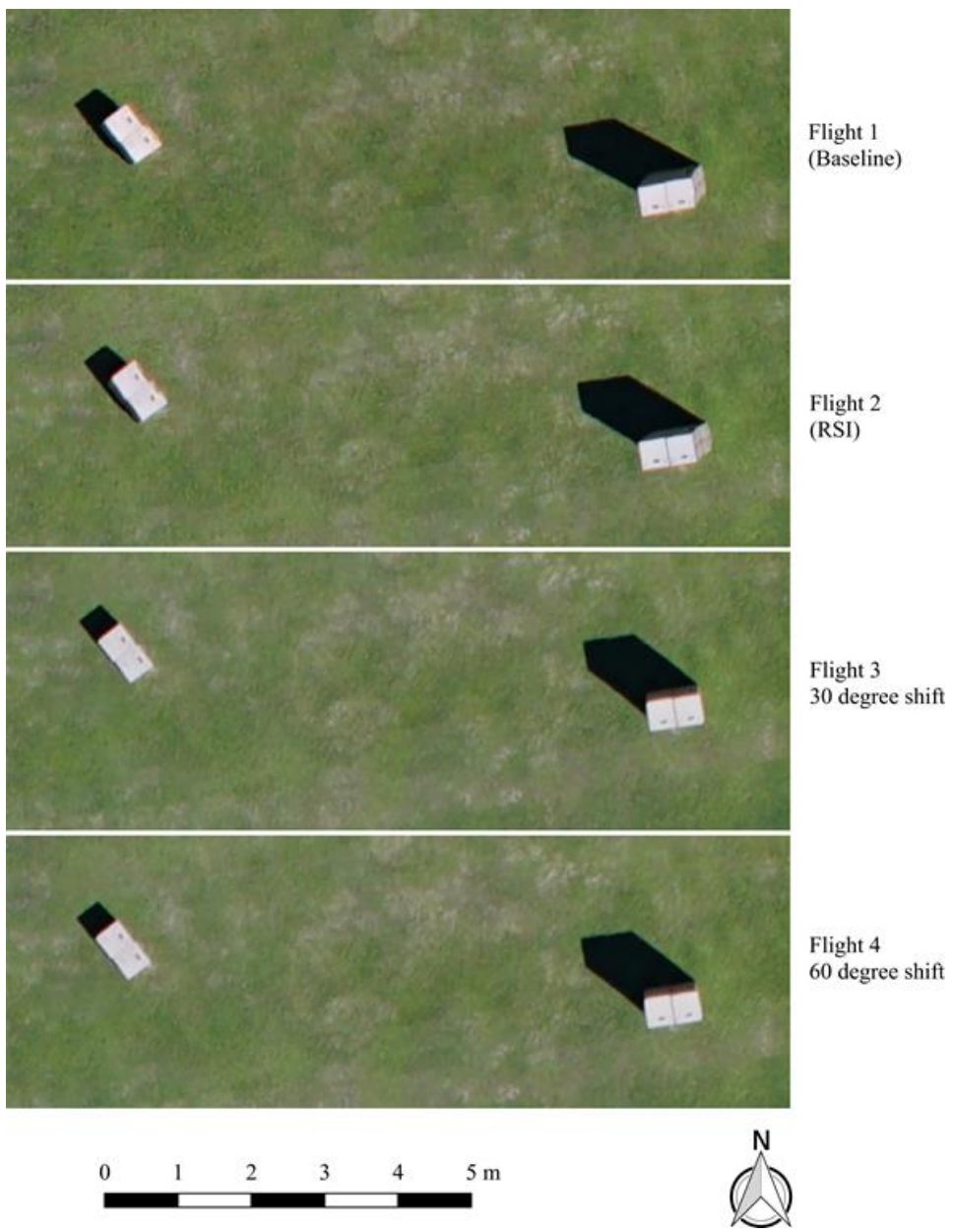

Figure 4: Reproducible Parallax with RSI. Shows differences in appearance of lean with variations in view geometries

\section{SFM image processing}

Structure-from-motion image processing was conducted identically for each flight using the Agisoft Photoscan workflow. Within Photoscan, four 'chunks', or groups of images, were created (one chunk per flight). Images for each flight were loaded into their respective chunks and the PPK image locations were loaded from the Bramor's logfile as reference data.

Six ground control points collected with the PPK rover surveying unit were then loaded and visually identified in the imagery for each of the chunks/flights. Image alignment was performed on each flight. The low-accuracy tie points were discarded as follows: 1) points with a reconstruction uncertainty greater than or equal to $10 \%$;2) points with reprojection errors greater than $1 \%$; 3 ) points with projection errors greater than or equal to $10 \%$. After these tie points were discarded, the image alignments were optimized. 
To limit variations in DSM accuracy only to variations in image station location, all variables were kept identical in the workflow. The sparse cloud was set to an unlimited number of points for each of the four flights. The 'Ultra-high Quality' dense point cloud setting was chosen for creating the point cloud. Repeated observations have demonstrated that this setting allows for the creation of DSMs with ground sample distances identical to those of the orthomosaics. The point cloud densities for each of the four flights averaged 2,610 points per $\mathrm{m}^{2}$.

DSMs and orthomosaics were exported and saved for analysis using the European Terrestrial Reference System (ETRS89) and the Lambert Azimuthal Equal Area projection (ETRS-LAEA). This projection is recommended for 'statistical analysis and display' (Annoni et al., 2001).

\section{Analysis}

Vertical and volumetric change estimation was performed using the DSMs as described in Section "SFM image processing", and an accuracy assessment of the results was performed using known volumetric change values.

\section{Measurement of Volumetric Change}

Change detection can be performed using various methods, such as image differencing, direct object measurements, and hybrid approaches (Chen et al., 2012; Song et al., 2001; Stow, 1999). Accurate DSM registration between the initial and subsequent flights is crucial for pixel-differencing methods (Stow, 1999). The image frames collected had a nominal GSD of $1.96 \mathrm{~cm}$, and the resultant GSD of the DSMs was $1.96 \mathrm{~cm}$. The post-processed image station locations and the ground control points were used for absolute positional registration of each DSM during the SFM processing phase described in Section "SFM image processing". Additional co-registration between the DSMs of the four flights was not performed.

Image differencing was used as the change detection method. Measurements of the changes were performed in GIS using Quantum GIS and ERDAS IMAGINE.

DSMs consist of pixels, where each pixel occupies an area and its value represents its height. Multiplication of height by the pixel dimensions yields the volume measurement of a pixel relative to sea level. Volumetric change was measured by differencing the baseline DSM with the DSM from subsequent passes, resulting in the value of pixels being the change in height between observations. When multiplied by the pixel dimensions, this results in an estimate of volumetric change.

ERDAS IMAGINE was used to perform the image differencing and to create polygon features from clumps of pixels that experienced $0.010 \mathrm{~m}$ or more of vertical change. These polygons were used to represent the boundaries of the stacks of boxes that were manipulated. The raster calculator tool in Quantum GIS was used to calculate the change volumes from the differenced DSMs, and zonal statistics were applied to the polygons to calculate the measured volumetric changes for each stack of boxes. 


\section{Test of consistency}

The seven stacks of boxes that were unchanged during the flights produced measurable variations in modelled height between the baseline and subsequent acquisitions. These height variations of invariant features in the differenced DSMs are therefore errors, and these errors are hypothesized to be more consistent between the baseline and RSI acquisition than between the baseline and non-RSI acquisitions.

To measure the difference in modelled height between flights, the centre-pixel of each stack of boxes was selected and a $10 \mathrm{~cm}$ radius buffer created. Mean height values were then calculated for each buffer area and subtracted from their known height values. The difference between the modelled and known heights for invariant features represents an estimate of the errors between paired image sets.

The RMSE and standard deviation of errors across the seven stacks of boxes was calculated for each of the three non-baseline flights. The RMSE is used for calibrating the change detection results, and the standard deviations are used to compare the consistency of the errors to invariant features with the RSI versus non-RSI methods.

\section{Calibrating change detection results}

Calibration of measured vertical changes was performed by adding the RMSE height values from the unchanged box stacks to the height values of the stacks of boxes where negative changes were detected (i.e., boxes were removed) and subtracting the RMSE height values from the stacks where positive change values were detected (i.e., boxes were added). For all post-change flights, these calibrations used the precisely measured heights of the boxes. Once the height values were adjusted, volumes were re-calculated.

\section{Results}

Results are presented for the uncalibrated volumetric changes, calibrated volumetric changes, and the height errors of the invariant stacks of boxes used for calibration.

Overall, RSI had substantially lower uncalibrated volumetric change measurement errors for five of the six stacks of boxes, $1 \mathrm{~A}$ to $6 \mathrm{~A}$, that existed during the baseline flight and were manipulated. RSI had generally greater errors than the non-RSI flights for the stacks of boxes $8 \mathrm{~A}-13 \mathrm{~A}$, which were added after the baseline flight, where originally there was just a grass. Figure 5 shows the uncalibrated percent errors for each stack of boxes, by flight. For boxes $5 \mathrm{~A}$ and $8 \mathrm{~A}-13 \mathrm{~A}$, no repeated geometry views were possible for the RSI flight, as the boxes did not exist in the baseline but did exist in subsequent flights. Furthermore, estimates of the surface height for the grassy field and subsequent volume measurement of the new boxes would have been based solely on the quality of the view perspectives for each individual flight, rather than the correlation of views between flights. This may explain why RSI did not perform as well; it may have had less complete or useful views of the new boxes. 


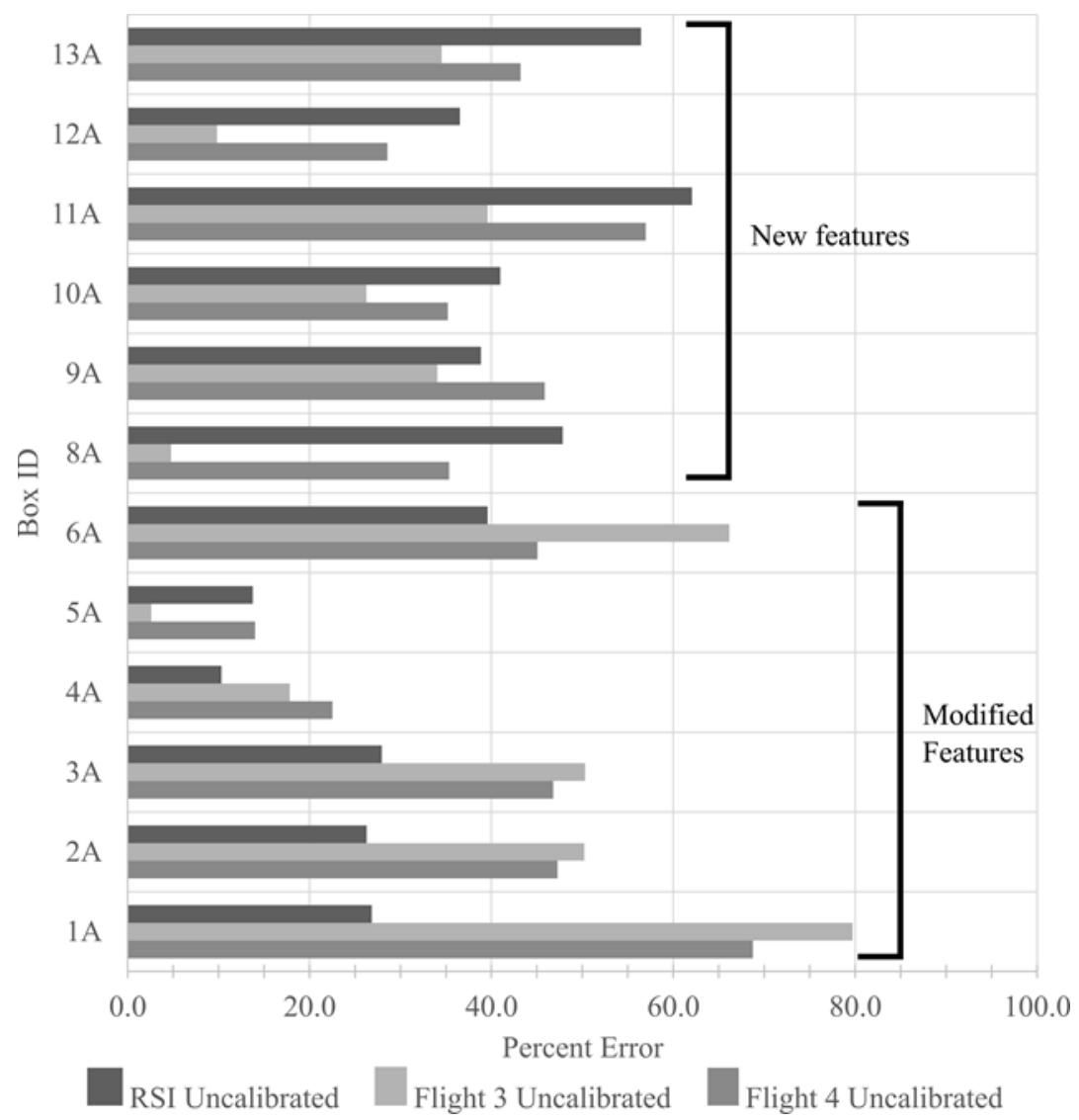

Figure 5: Uncalibrated Volumetric Percent Errors. Shows the percent error by box stack ID. 'A' indicates that the box was manipulated. Box 7 was an invariant feature, so it does not appear here. New features (i.e. those that did not exist in the baseline flight) are boxes 8A to 13A; modified features (i.e. those that existed in the baseline flight) are boxes 1A to 6A

Results of calibrating the errors for each acquisition show that RSI produced greater improvement to the accuracy of each volumetric measurement and to the overall accuracy. Figure 6 shows the calibrated percent errors for each stack of boxes, by flight. The results in Figure 6 indicate that the RSI measurements are more comparable to the non-RSI measurements for the new boxes, $8 \mathrm{~A}$ to $13 \mathrm{~A}$, after calibration. Furthermore, the results show that for the boxes that existed in the baseline and RSI flights, $1 \mathrm{~A}$ to $4 \mathrm{~A}$, the improvement over non-RSI is substantial.

Table 1 provides the individual errors of stacks, the RMSE, MPE and standard deviations. As seen in Figure 5, Figure 6 and Table 1, there is a clear difference in the RMSE, MPE and standard deviations of boxes $1 \mathrm{~A}$ to $6 \mathrm{~A}$ compared with $8 \mathrm{~A}$ to $13 \mathrm{~A}$. This implies that RSI yielded higher accuracy with persistent objects that experienced volumetric change compared with objects that are newly added to a scene. Box $5 \mathrm{~A}$, while it existed in the baseline flight, was removed completely for the subsequent flights. Therefore, it more closely resembles the error results for boxes $8 \mathrm{~A}$ to $13 \mathrm{~A}$. The standard deviation of error for all groups of box 
stacks and all box stacks combined was lower for the RSI approach, indicating that the errors were more systematic between the baseline and the RSI flights than between the baseline and the non-RSI flights.

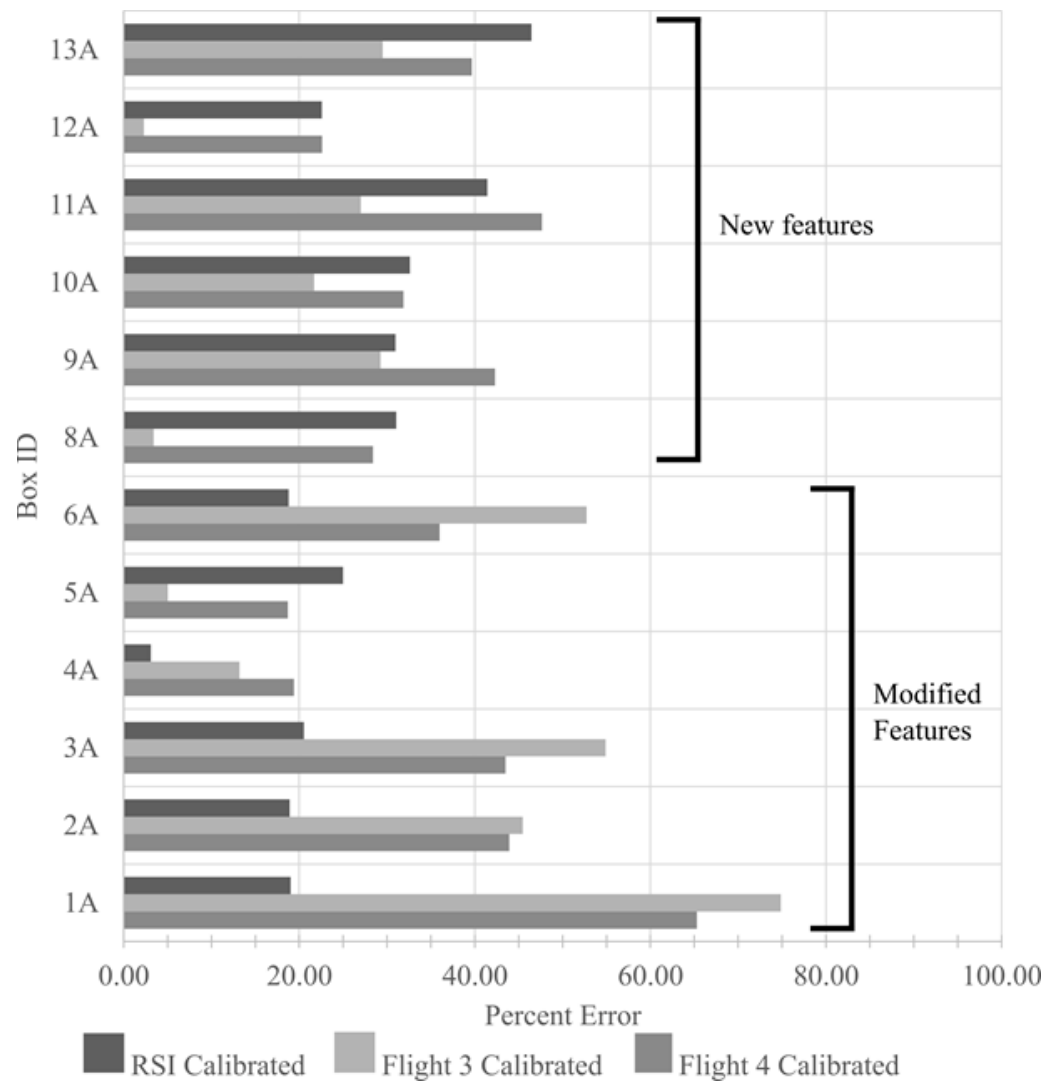

Figure 6: Calibrated Volumetric Percent Errors. Shows the percent error by box stack ID. 'A' indicates that the box was manipulated. Box 7 was an invariant feature, so it does not appear here. New features (i.e. that did not exist in the baseline flight) are boxes $8 \mathrm{~A}$ to 13A; modified features (i.e. that existed in the baseline flight) are boxes $1 \mathrm{~A}$ to $6 \mathrm{~A}$ 
Table 1: Volumetric Change Errors and Assessments. Shows the individual measurement and percent errors by box stack and flight, the RMSE, MPE and SD for boxes that were manipulated and those newly placed, and for all boxes combined

\begin{tabular}{|c|c|c|c|c|c|c|c|c|}
\hline Box ID & $\begin{array}{l}\text { Initial } \\
\text { Volume } \\
\left(\mathrm{m}^{3}\right)\end{array}$ & $\begin{array}{l}\text { Volume } \\
\text { Change } \\
\left(\mathrm{m}^{3}\right)\end{array}$ & $\begin{array}{l}\text { RSI } \\
\text { Error } \\
\left(\mathrm{m}^{3}\right)\end{array}$ & $\begin{array}{l}\text { RSI } \\
\text { PE }\end{array}$ & $\begin{array}{l}\text { Flight } \\
3 \text { Error } \\
\left(\mathrm{m}^{3}\right)\end{array}$ & $\begin{array}{l}\text { Flight } \\
3 \text { PE }\end{array}$ & $\begin{array}{l}\text { Flight } 4 \\
\text { Error } \\
\left(\mathrm{m}^{3}\right)\end{array}$ & $\begin{array}{l}\text { Flight } \\
4 \text { PE }\end{array}$ \\
\hline $1 \mathrm{~A}$ & 0.710 & -0.178 & -0.034 & 19.0 & -0.133 & 74.9 & -0.116 & 65.3 \\
\hline $2 \mathrm{~A}$ & 0.532 & -0.178 & -0.034 & 18.9 & -0.081 & 45.5 & -0.078 & 43.9 \\
\hline $3 \mathrm{~A}$ & 0.355 & -0.178 & -0.036 & 20.5 & -0.097 & 54.9 & -0.077 & 43.5 \\
\hline $4 \mathrm{~A}$ & 0.890 & -0.089 & -0.003 & 3.1 & -0.012 & 13.2 & -0.017 & 19.4 \\
\hline $5 \mathrm{~A}$ & 0.177 & -0.089 & 0.022 & 25.0 & 0.005 & 5.0 & 0.0170 & 18.7 \\
\hline $6 \mathrm{~A}$ & 0.043 & -0.043 & -0.008 & 18.8 & -0.023 & 52.7 & -0.016 & 36.0 \\
\hline $\begin{array}{l}\text { RMSE }\left(\mathrm{m}^{3}\right) \text {, } \\
1 \mathrm{~A}-6 \mathrm{~A}\end{array}$ & & & 0.026 & & 0.076 & & 0.066 & \\
\hline MPE, 1A-6A & & & & 17.6 & & 41.0 & & 37.8 \\
\hline SD, 1A-6A & & & 0.021 & & 0.050 & & 0.046 & \\
\hline \multicolumn{9}{|l|}{ Box ID } \\
\hline $8 \mathrm{~A}$ & 0.089 & 0.089 & 0.028 & 31.0 & -0.003 & 3.4 & 0.025 & 28.4 \\
\hline $9 \mathrm{~A}$ & 0.178 & 0.178 & 0.055 & 31.0 & 0.052 & 29.3 & 0.075 & 42.3 \\
\hline $10 \mathrm{~A}$ & 0.178 & 0.178 & 0.058 & 32.6 & 0.039 & 21.7 & 0.057 & 31.9 \\
\hline $11 \mathrm{~A}$ & 0.043 & 0.043 & 0.018 & 41.4 & 0.012 & 27.0 & 0.021 & 47.7 \\
\hline $12 \mathrm{~A}$ & 0.178 & 0.178 & 0.040 & 22.6 & 0.004 & 2.3 & 0.040 & 22.6 \\
\hline $13 \mathrm{~A}$ & 0.089 & 0.089 & 0.041 & 46.5 & 0.026 & 29.5 & 0.035 & 39.7 \\
\hline $\begin{array}{l}\text { RMSE }\left(\mathrm{m}^{3}\right) \text {, } \\
\text { 8A-13A }\end{array}$ & & & 0.042 & & 0.029 & & 0.046 & \\
\hline MPE, 8A-13A & & & & 34.2 & & 18.9 & & 35.4 \\
\hline $\mathrm{SD}, 8 \mathrm{~A}-13 \mathrm{~A}$ & & & 0.014 & & 0.019 & & 0.019 & \\
\hline $\begin{array}{l}\text { Total RMSE } \\
\left(\mathrm{m}^{3}\right)\end{array}$ & & & 0.035 & & 0.057 & & 0.057 & \\
\hline Total MPE & & & & 25.9 & & 30.0 & & 36.6 \\
\hline Total SD & & & 0.033 & & 0.055 & & 0.057 & \\
\hline
\end{tabular}

The results of the vertical error analysis of the unchanged box stacks, $1 \mathrm{~B}$ to $7 \mathrm{~B}$, that were used for the calibration of the changed box stacks are shown in Table 3. The results in Table 2 indicate that while the absolute error measurements and the RMSE were higher for the RSI flight, the standard deviation of the invariant boxes' errors in the RSI flight were lower than for the non-RSI flights. The lower standard deviation is an indication that the RMSE could be, and was, used as a calibration of the measured change values. 
Table 2: Changes in height of invariant features recorded as errors

\begin{tabular}{|l|l|l|l|l|l|}
\hline Box ID & $\begin{array}{l}\text { Initial } \\
\text { Height } \\
\mathbf{( m )}\end{array}$ & $\begin{array}{l}\text { Actual } \\
\text { Change } \\
\mathbf{( m )}\end{array}$ & $\begin{array}{l}\text { RSI } \\
\text { Error } \\
\mathbf{( m )}\end{array}$ & $\begin{array}{l}\text { Flight 3 } \\
\text { Error } \\
\mathbf{( m )}\end{array}$ & $\begin{array}{l}\text { Flight 4 } \\
\text { Error } \\
\mathbf{( m )}\end{array}$ \\
\hline 1B & 2.636 & 0.000 & -0.037 & 0.031 & -0.019 \\
\hline 2B & 1.977 & 0.000 & -0.037 & 0.012 & -0.019 \\
\hline 3B & 1.318 & 0.000 & -0.033 & 0.017 & -0.012 \\
\hline 4B & 0.659 & 0.000 & -0.032 & 0.018 & -0.008 \\
\hline 5B & 0.382 & 0.000 & -0.032 & 0.036 & 0.012 \\
\hline 6B & 0.282 & 0.000 & -0.045 & -0.014 & -0.026 \\
\hline 7B & 0.380 & 0.000 & -0.028 & 0.006 & 0.002 \\
\hline RMSE & & & $\mathbf{0 . 0 3 5}$ & $\mathbf{0 . 0 2 2}$ & $\mathbf{0 . 0 1 6}$ \\
\hline SD & & & $\mathbf{0 . 0 0 5}$ & $\mathbf{0 . 0 1 5}$ & $\mathbf{0 . 0 1 2}$ \\
\hline
\end{tabular}

The statistical significance of the mean of differences for each stack of boxes by flight/acquisition method was tested using paired t-tests, which is appropriate in this case as the same changes to the stacks of boxes were measured three times. The results of the tests are presented in Table 3 . At a p-value of 0.05 , the results show significant difference between the errors reported for RSI and Flight 3 among changed boxes, new boxes, and overall. For RSI and Flight 4, there is significant difference for the changed boxes only. Between Flight 3 and Flight 4, there is significant difference in the new boxes and overall. These results show no statistical significance in the difference between RSI and Flight 4 among the new boxes, or overall, and no statistical significance in the difference between Flight 3 and Flight 4 among the changed boxes.

Table 2: Paired t-test of the significance of difference between mean errors per flight. $M_{\text {diff }}=$ mean of the differences, $\mathrm{df}=$ degrees of freedom, ${ }^{*}=$ significant at the 0.05 level

\begin{tabular}{|l|l|l|l|l|}
\hline Paired t-test & $\mathbf{M}_{\text {diff }}$ & t-value & df & p-value \\
\hline RSI and Flight - changed boxes & 0.0414 & 2.912 & 5 & $0.03331^{*}$ \\
\hline RSI and Flight $3-$ new boxes & 0.0184 & 3.442 & 5 & $0.01839^{*}$ \\
\hline RSI and Flight $3-$ all boxes & 0.0299 & 3.721 & 11 & $0.00338^{*}$ \\
\hline RSI and Flight $4-$ changed boxes & 0.0325 & 2.695 & 5 & $0.04304^{*}$ \\
\hline RSI and Flight $4-$ new boxes & -0.0022 & -0.584 & 5 & 0.58460 \\
\hline RSI and Flight $4-$ all boxes & 0.0151 & 1.898 & 11 & 0.08425 \\
\hline Flight 3 and Flight $4-$ changed boxes & -0.0090 & -2.312 & 5 & 0.06873 \\
\hline Flight 3 and Flight $4-$ new boxes & -0.0206 & -4.681 & 5 & $0.00543^{*}$ \\
\hline Flight 3 and Flight $4-$ all boxes & -0.0148 & -4.480 & 11 & $0.00093^{*}$ \\
\hline
\end{tabular}




\section{Discussion}

RSI has previously been shown to offer higher accuracy for two-dimensional image change detection by enabling precisely co-registered multi-temporal image pairs (Coulter et al., 2003; Stow, 1999). Applying and assessing the RSI acquisition method for use in DSM generation and volumetric change estimation had not previously been conducted.

The change detection results clearly show that RSI enables improved accuracy of volumetric change measurements of vertical objects that experience changes (e.g., partially removed pile, damaged building), and that RSI can achieve comparable results when the volumes to be measured are for newly placed objects. The potential sources of error leading to the differences between the accuracies of changed versus new objects, and uncalibrated versus calibrated objects are discussed in this section.

RSI in this research is expected to better replicate the differential parallax of individual camera stations of a baseline image acquisition. Differential view geometries of images with vertical features result in a given amount of parallax and pattern of tie points extracted in the baseline flight. If the RSI flight then perfectly replicated the view geometries of the individual camera stations in the baseline flight, we would expect the time- $n$ flight to have similar patterns of errors for vertical features. It is believed that the higher overall volumetric change detection accuracy in both the uncalibrated and calibrated measurements for boxes $1 \mathrm{~A}$ to $6 \mathrm{~A}$ shows that the similar view geometries of the camera stations of the RSI and baseline flights resulted in similar errors between time- 1 and time- $n$ flights, which were therefore partially normalized during image differencing.

We also find that the accuracy of the RSI method for newly placed features is comparable to the accuracy of newly placed features with the non-RSI method. Table 3 shows no statistical significance to the difference in errors between RSI and Flight 4 for the new boxes, and it does show a significance to the difference in errors between RSI and Flight 3 for the new boxes. The MPE of RSI is 34.17 for the new boxes, 18.87 for the new boxes in Flight 3, and 35.42 for the new boxes in Flight 4. Unlike for the changed boxes and overall results, the results of the new boxes show variation in the performance of RSI versus non-RSI, and variations between non-RSI at 30 degrees flight-line rotation and non-RSI at 60 degrees flight-line rotation. As the newly placed boxes were not present in the baseline imagery, normalization of the systematic errors in the differential parallax resulting from repeating the camera station locations could not be replicated between the baseline and RSI flights for those boxes. Furthermore, as the generation of tie points between images in SFM is impacted by the actual view geometries from different camera station locations, it is plausible that for a given direction and location of a flight line, more oblique view geometries and/or better overlap/sidelap may be acquired for some objects. We hypothesize this to be the cause for the variability in accuracies of newly placed features among the flights.

To ensure that the per-flight automatically-generated camera models in the SFM application were not a contributing factor to the findings, the results were repeated by utilizing the camera model parameters of the baseline flight across all flights. This resulted in increased volumetric change errors for every box stack and for every flight. 
The geographic coordinates of image stations were recorded in flight with Bramor's DGPS receiver and post-processed to a three-dimensional, absolute positional accuracy of $0.015 \mathrm{~m}$. The positional accuracy of the ground control points is also $0.015 \mathrm{~m}$. At hyper-spatial GSDs like those employed in this study, these small positional errors also contribute to the overall vertical and volume accuracy measurements. Use of an equal area projection was found to be critical to the accuracy of volumetric measurements.

\section{Conclusion}

RSI is a useful acquisition method for achieving higher volumetric change accuracies with SFM. This was demonstrated using a fixed-wing UAS and off-the-shelf digital camera, and four imagery acquisitions performed over an area where artificial changes were introduced. The RSI method resulted in an overall, calibrated, volumetric MPE of $25.9 \%$ compared to the other flights with $30.0 \% \mathrm{MPE}$ and $36.6 \% \mathrm{MPE}$. Where the purpose of change detection is to measure volumetric changes to extant features, the calibrated RSI method resulted in an MPE of $17.6 \%$ compared to a $41.0 \% \mathrm{MPE}$ and $37.8 \% \mathrm{MPE}$ for the non-RSI methods. Further reduction of errors for all measurements could potentially be achieved with greater sidelap and overlap of images during the acquisition, and a lower nominal ground sample distance. Improvements to the calibration of measurements for the RSI method could be achieved by better repeating the view geometries, which would result in a repeat camera station location of better than $4.22 \mathrm{~m}$ RMSE.

\section{Acknowledgements}

This research project was sponsored by the Austrian Marshall Plan Foundation through their research and cultural exchange Fellowship. We would like to thank the Marshall Plan Foundation for funding this research, the generation of knowledge, and cultural exchange. This work was also partially funded by the National Science Foundation Directorate of Engineering, Infrastructure Management and Extreme Events (IMEE) program (Grant Number G00010529). 


\section{References}

Annoni, A., Luzet, C., Gubler, E., Ihde, J. (2001). Map Projections for Europe. Inst. Environ. Sustain. 1131.

C-Astral, (2015). BRAMOR ppX [WWW Document]. URL http://www.castral.com/en/products/bramor-ppx/ (accessed 3.14.17).

Chen, G., Hay, G.J., Carvalho, L.M.T., Wulder, M.A., (2012). Object-based change detection 33, 4434-4457.

Coulter, L.L., Stow, D. a., Baer, S., (2003). A frame center matching technique for precise registration of multitemporal airborne frame imagery. IEEE Trans. Geosci. Remote Sens. 41, 2436-2444. https://doi.org/10.1109/TGRS.2003.819191

Jensen, J.R., Im, J., (2007). Remote sensing change detection in urban environments, in: Geo-Spatial Technologies in Urban Environments. Springer, pp. 7-31.

Lee, C.Y., Jones, S.D., Bellman, C.J., Buxton, L., (2008). Dem Creation of a Snow Covered Surface Using Digital Aerial Photography 831-836.

Lippitt, C.D., Stow, D., Coulter, L., (2015). Time-Sensitive Remote Sensing. Springer, New York.

Niethammer, U., James, M.R., Rothmund, S., Travelletti, J., Joswig, M., (2012). UAV-based remote sensing of the Super-Sauze landslide: Evaluation and results. Eng. Geol. 128, 2-11. https://doi.org/10.1016/j.enggeo.2011.03.012

Rosa, M.F., Stow, D.A., (2014). Mapping fuels at the wildland-urban interface using colour orthoimages and LiDAR data. Geocarto Int. 29, 570-588. https://doi.org/10.1080/10106049.2013.819040

Slama, C.C., Theurer, C., Henriksen, S.W., (1980). Manual of photogrammetry. American Society of photogrammetry.

Song, C., Woodcock, C.E., Seto, K.C., Lenney, M.P., Macomber, S.A., (2001). Classification and change detection using Landsat TM data: When and how to correct atmospheric effects? Remote Sens. Environ. 75, 230-244. https://doi.org/10.1016/S0034-4257(00)00169-3

Stow, D.A., (1999). Reducing the effect of misregistration on pixel level change detection. Int. J. Remote Sens.

Stow, D.A., Coulter, L.C., MacDonald, G., Lippitt, C.D., McCreight, R., Zamora, N., (2016). Evaluation of Geometric Elements of Repeat Station Imaging and Registration. Photogramm. Eng. Remote Sens. 82, 775-788.

Turner, D., Lucieer, A., Watson, C., (2012). An Automated Technique for Generating Georectified Mosaics from Ultra-High Resolution Unmanned Aerial Vehicle (UAV) Imagery, Based on Structure from Motion (SfM) Point Clouds. Remote Sens. 4, 1392-1410. https://doi.org/10.3390/rs4051392

Westoby, M.J., Brasington, J., Glasser, N.F., Hambrey, M.J., Reynolds, J.M., (2012). ' Structure-fromMotion' photogrammetry: A low-cost, effective tool for geoscience applications. Geomorphology 179, 300-314. https://doi.org/10.1016/j.geomorph.2012.08.021

Zhang, S., Lippitt, C.D., Bogus, S.M., Loerch, A.C., Sturm, J.O., (2016). The accuracy of aerial triangulation products automatically generated from hyper-spatial resolution digital aerial photography. Remote Sens. Lett. 7, 160-169. https://doi.org/10.1080/2150704X.2015.1121299

Zhang, Y., Xiong, J., Hao, L., (2011). Photogrammetric processing of low-altitude images acquired by unpiloted aerial vehicles. Photogramm. Rec. 26, 190-211. https://doi.org/10.1111/j.1477-

9730.2011.00641.x 